\title{
Use of a gray level co-occurrence matrix to characterize duplex stainless steel phases microstructure
}

\author{
F. R. Renzetti, L. Zortea \\ Università di Roma "La Sapienza", D.I.C.M.A. via Eudossiana, 18 - Roma
}

\begin{abstract}
Duplex stainless steels are widely used in industry. This is due to their higher strength compared to austenitic steels and to their higher toughness than ferritic steels. They also have good weldability and high resistance to stress corrosion cracking.

These steels are characterized by two-phase microstructures composed by almost the same level of ferrite and austenite.

Duplex steel 2205 samples evaluated are: as received, cold rolled $(33 \%)$ and heat-treated at $800^{\circ} \mathrm{C}$ for 10 hours. A metallographic etching with $10 \%$ oxalic acid has been carried out to highlight the phases morphology. Some photos have been taken by SEM microscope and submitted to image analysis.

The analysis carried out is based on the determination of co-occurrence matrix and on the following interpretation of appropriate indicators. Through these indicators is possible to estimate the features of images objectively.
\end{abstract}

KEYwORDS. Gray level co-occurrence matrix (GLCM); Image analysis; Duplex stainless steel; Microstructure characterization.

\section{INTRODUCTION}

$\mathrm{D}$ uplex stainless steels are resistant to intergranular corrosion [1]. They are characterized by almost the same amount of two phases, ferrite and austenite.

In the pseudo binary phase diagram of DSS, we can see after a first phase of fully ferritic solidification, there is a partial conversion of the microstructure in austenitic phase [2].

In this paper a non invasive methodology is used to highlight the DSS microstructure, an algorithm based on a statistical approach, which allowed an objective and repeatable study of some images obtained by electron microscope.

The statistical approach for image analysis based on the matrix of co-occurrence (GLCM Gray Level Co-occurrence Matrix) is widespread in many fields, alone or synergistically with other analysis, to evaluate the images morphology. This one, better known as "texture" (an innate property of all the virtual surfaces), gives information on the disposition of the structures and their relations with the environment.

Relevant indicators will be derived from the GLCM to describe the "texture" of the image.

Through this method it's possible to see tool marks, the presence of surface defects and also the grain boundaries of each phase. 


\section{ANALYSIS METHOD}

he spatial relationship of gray levels is expressed by the co-occurrence matrix suggested by Haralick [3, 4] in the 70’s.

GLCM method assumes that the information on the texture image is adequately provided by a particular matrix.

The technique is based on the manipulation of the gray levels of an image. If we consider a digital color image (Fig.1) the first step is to convert it in gray tones (Fig.2) using any image viewer.

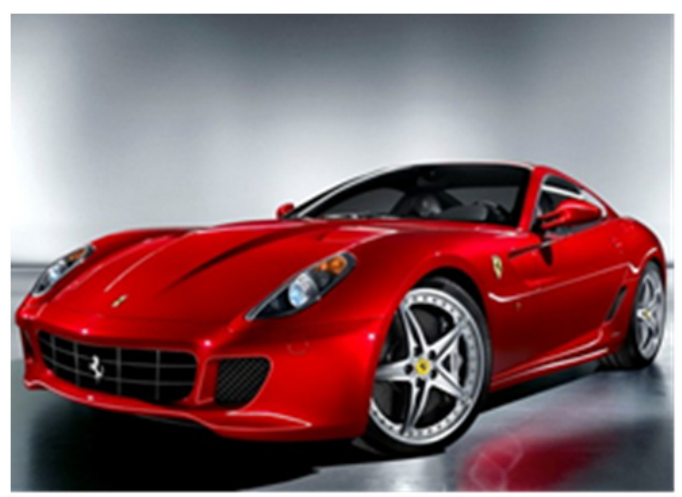

Figure 1: Digital color image.

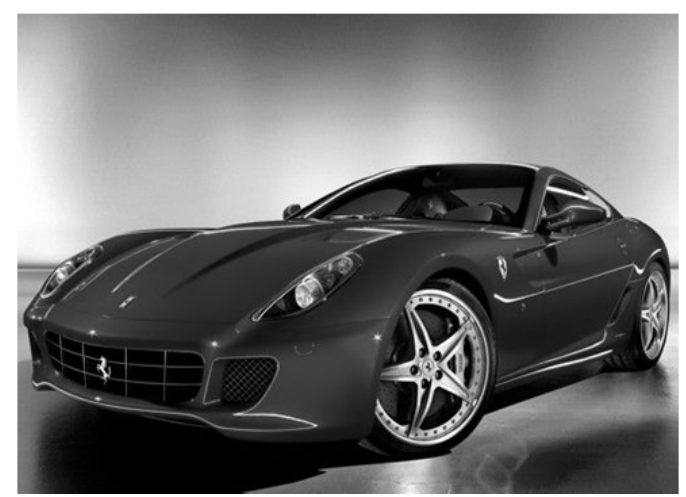

Figure 2: Gray tones image (256 levels).

Digital images are made up of pixels that are stored in memory using 8 bit because it is assumed that $2^{8}=256$ gray levels are sufficient to describe the nuances noticeable to the human eye. The gray levels are distinguished with numbers ranging from 0 (black) to 255 (white).

Each gray level is stored as a binary number or as a sequence of bits. Each number corresponds to different gray level or different shades of gray level.

A particular shade of gray is then associated to each pixel component.

The transformation in 256 gray levels is common with any Image Analysis program.

Number of gray levels is an important factor in GLCM computation. More levels would mean more accurate textural information, but also an increase in computational costs.

A procedure of segmentation reduces the shades of gray. The pictures below show the difference between an image in 64 shades of gray (Fig.3) and one in 8 (Fig.4):

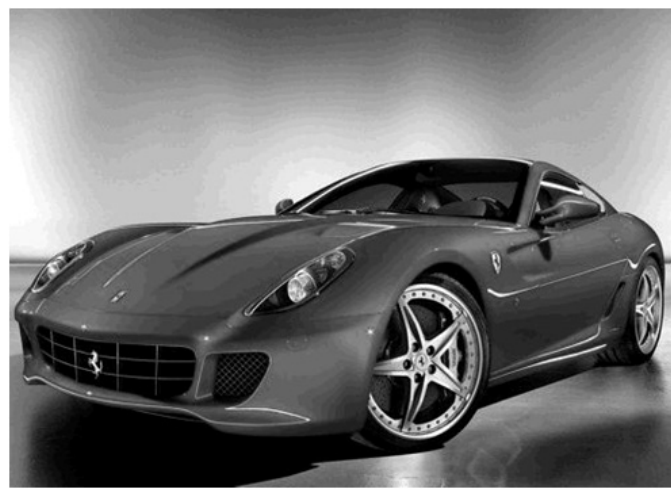

Figure 3: Gray tones image (64 levels)

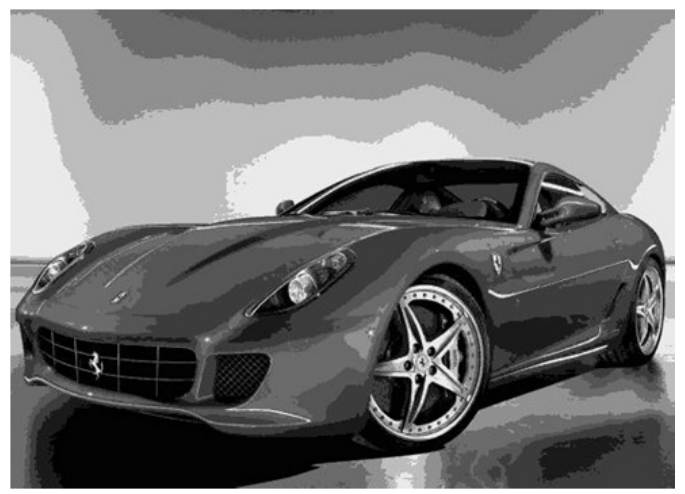

Figure 4: Gray tones image (8 levels)

In this article has been implemented a Matlab ${ }^{\circledR}$ program that carried out the conversion of the used images in shades of gray, and subsequently reduced the levels of them.

In particular we will compare a performance at 8 and 64 levels of gray.

The goal is to be able to recognize the "objects" that make up the image, i.e. phases, grain boundaries, inclusions, defects, etc.

Below the construction of the GLCM [5] matrix is shown with a simple example. 
The GLCM is a square matrix whose size is equal to the number of gray levels which the starting image has been reduced in.

Consider a gray tones image, let us take a small part, for example $4 x 4$ pixels. As already mentioned, each pixel has a gray tone (Fig.5).

In Fig.6 there is the corresponding matrix of image, where shades of gray have been replaced by the corresponding number on the grayscale.

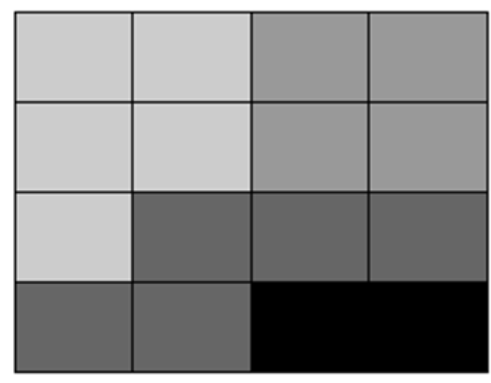

Figure 5: Matrix 4x4 pixels in 4 shades of gray.

\begin{tabular}{|c|c|c|c|}
\hline 192 & 192 & 150 & 150 \\
\hline 192 & 192 & 150 & 150 \\
\hline 192 & 128 & 128 & 128 \\
\hline 128 & 128 & 0 & 0 \\
\hline
\end{tabular}

Figure 6: Matrix of image.

GLCM of an image is computed using a displacement vector d, defined by its radius $\delta$ and orientation $\theta$ [3].

The technique works by forming a "detection window" on the image that scrolls over it. The window size and directions will vary depending on the problem at hand.

The choice of $\delta$ often is in the range of values 1 and 2. Indeed, it is easy to see that the probability that two pixels have the same gray level is greater the more they are close.

Small values of $\delta$ are used to better analyze fine textures, grain boundaries, the presence of carbides or nitrides, the remains of alumina powder used for polishing the specimen that has been not completely removed.

Regarding the choice of $\theta$ we know that each pixel has 8 neighbours at $\theta=0^{\circ}, 45^{\circ}, 90^{\circ}, 135^{\circ}, 180^{\circ}, 225^{\circ}, 270^{\circ}, 315^{\circ}$ but choose the neighbour at $\theta=0^{\circ}$ or at $\theta=180^{\circ}$ is similar for the GLCM definition. So the choice may fall to 4 neighbouring pixels at $\theta=0^{\circ}, 45^{\circ}, 90^{\circ}$ and $135^{\circ}$ : horizontal, right diagonal, vertical and left diagonal.

In the example $\delta=1$ and $\theta=0^{\circ}$ are chosen. Then the way is from left to right in horizontal with one pixel at one time.

Three parameters will be considered to describe an image through GLCM: the number of gray levels, the orientation angle and the length of displacement. These parameters can be changed to improve the characterization.

The algorithm will start in the top left corner and count the occurrences of each reference pixel to neighbour pixel relationship.

Thus, each element $(i, j)$ of GLCM is the sum of the number of times that pixel value $i$ was located some distance $\delta$ and angle $\theta$ from pixel intensity $j$.

At the end of the process, the element $(i, j)$ represents how many times the gray levels $i$ and $j$ appears as a sequence of two pixels located at a defined distance $\delta$ along a chosen direction $\theta$.

GCLM can be defined as: "a two dimensional histogram of gray levels for a pair of pixels, which are separated by a fixed spatial relationship."

From the image above (Fig.6) it's obtained the following GLCM, Fig.7.

\begin{tabular}{|c|c|c|c|c|}
\hline & $1(0)$ & $2(128)$ & $3(150)$ & $4(192)$ \\
\hline $1(0)$ & 1 & 0 & 0 & 0 \\
\hline $2(128)$ & 1 & 3 & 0 & 0 \\
\hline $3(150)$ & 0 & 0 & 2 & 0 \\
\hline $4(192)$ & 0 & 1 & 2 & 2 \\
\hline
\end{tabular}

Figure 7: Initial configuration of the GLCM

\begin{tabular}{|c|c|c|c|c|}
\hline & 1 & 2 & 3 & 4 \\
\hline 1 & 0.083 & 0 & 0 & 0 \\
\hline 2 & 0.083 & 0.25 & 0 & 0 \\
\hline 3 & 0 & 0 & 0.166 & 0 \\
\hline 4 & 0 & 0.083 & 0.166 & 0.166 \\
\hline
\end{tabular}

Figure 8: Final configuration for the GLCM

Once the window of comparison ends scanning the image, the statistical measures begin to extract the characteristics of the matrix. 
The next step to determine the texture features is to express GLCM's terms as probabilities [6]; in order to achieve that goal selected statistics are applied by iterating through the matrix. The probability describes how often one gray tone will appear in a specified spatial relationship to another gray tone on the image. So the terms are divided in all possible combinations within the matrix of image along the selected direction.

Then it's considered the normalization equation whose formula follows:

$$
P(i, j)=\frac{C(i, j)}{\sum_{i, j=1}^{N} C(i, j)}
$$

where $\mathrm{C}(i, j)$ the value in cell $(i, j), \mathrm{P}(i, j)$ the probability, $\mathrm{N}$ the number of rows and columns. The final configuration of the co-occurrence matrix by 4 grey levels is shown in fig. 8 .

The properties of an image texture are detected indirectly by using the co-occurrence matrix from which special indexes called "image indicators" are explotated. The gray level co-occurrence matrix (GLCM [7]) is just the tool to start and then get the 14 indicators defined in the Haralick's theory.

The indicators calculated in this work are:

$$
\text { Entropy } \left.=-\sum_{i, j=1}^{N} P(i, j) \log P(i, j) \quad \text { (by convention if } \mathrm{P}(i, j)=0 \text { then } \log \mathrm{P}(i, j)=0\right)
$$

The Entropy indicator measures the disorder or complexity of an image.

The highest value of Entropy is found when the values of $\mathrm{P}(i, j)$ are allocated quite uniformly throughout the matrix. This happens when the image has no pairs of grey level, with particular preference over others. Entropy is strongly but inversely correlated to Energy.

$$
\text { Energy }=\sqrt{\sum_{i, j=1}^{N} P(i, j)^{2}}
$$

This statistic measures, the textural uniformity, it detects disorders in textures. This parameter indicates how much the texture is homogeneous, i.e. the GLCM contains values distributed fairly uniformly over all grid. It is high when the GLCM has few entries of large magnitude, low when all entries are almost equal. This is a measure of local homogeneity.

$$
\text { Contrast or inertia }=\sum_{i, j=1}^{N} P(i, j)(i-j)^{2}
$$

This statistic measures the difference between the highest and the lowest values of a contiguous set of pixels. It measures the amount of local variations present in the image. A low value of Contrast is obtained when the image has almost constant gray levels, vice versa this indicator presents high values for images.

$$
\text { Mutual information }=-\sum_{i, j=1}^{N} P(i, j) \log \left(\frac{P(i, j)}{P_{i}(i) P_{j}(j)}\right)
$$

where $P_{i}(i)=\sum_{i=1}^{N} P(i, j)$ and $P_{j}(j)=\sum_{j=1}^{N} P(i, j)$.

This indicator supplies further information by which the uncertainty about one variable is reduced by the given knowledge of the second variable.

\section{APPLICATION}

1 he duplex steel images examined have been obtained by the electron microscope.

Three samples of 2205 duplex steel were evaluated, one as received, one cold rolled (33\%), and one heat-treated at $800^{\circ} \mathrm{C}$ for 10 hours. 
To highlight the amount and morphology of the phases, an electrolytic etching in $10 \%$ acid oxalic solution was carried out. The image analysis was performed by defining a detection window smaller $(5 \times 6$ pixels $)$ than the original $(1000 \times 1200$ pixels). The parameters were calculated in detection windows arranged so as to cover the entire image, equally spaced in both horizontal and vertical directions.

For each position of the detection window the co-occurrence matrix was calculated for the image reduced to 64 and to 8 gray levels. In this way it can be observed how much important is the choice of the gray levels number.

\section{RESULTS}

Matlab® program has been developed to calculate the co-occurrence matrix and the indicators.

The image below (Fig. 9 ) shows an example of the output).

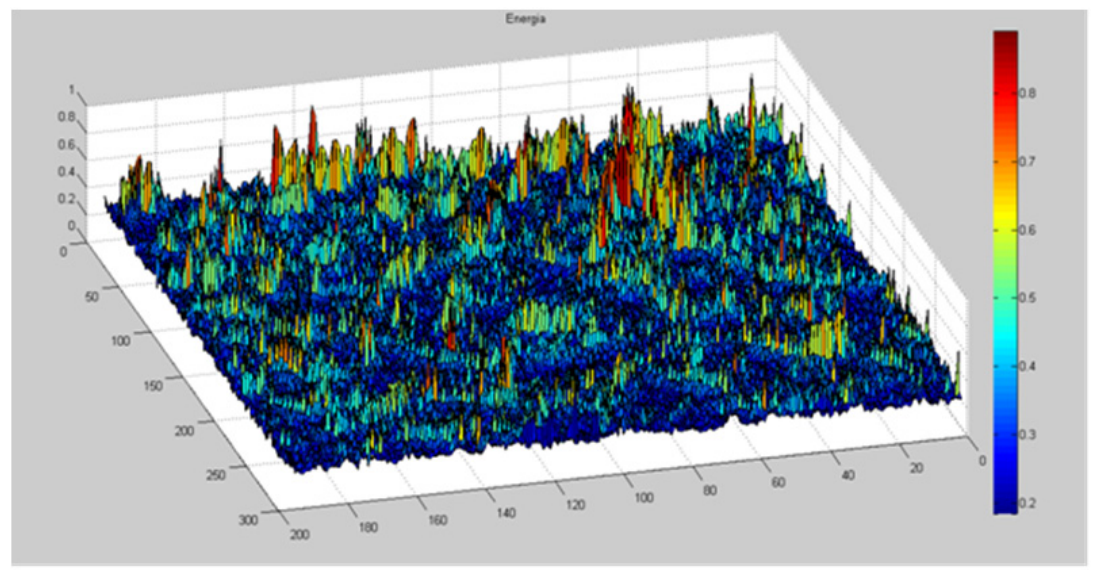

Figure 9: Energy indicator (8 gray tones) trend for the duplex as received.

Numerical values of the indicators are explicited in this way by the software. With an appropriate rotation of axes we get the mapping (Fig.10) of each indicator.

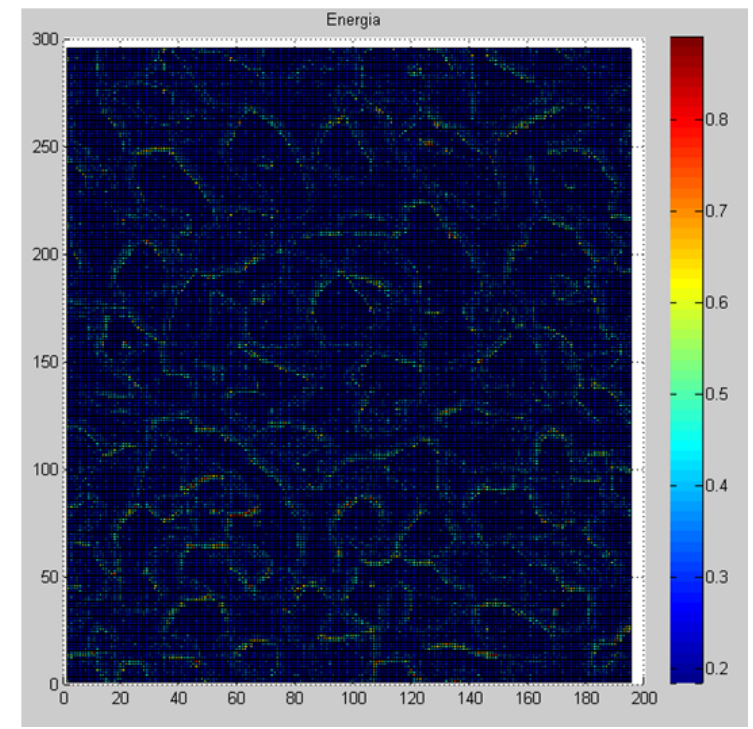

Figure 10: Energy indicator map.

The results provided by the chosen indicators show a good ability to describe the duplex steel "texture", showing in this case the grain boundaries, geminates and any impurities present in the sample.

The following images are obtained with the used algorithm. 
The tables below the group of pictures show the minimum and maximum values obtained for each indicator with 8 to 64 shades of gray.

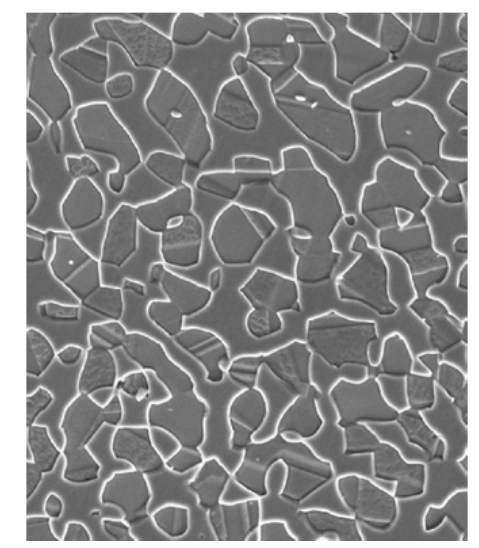

Figure 11: Duplex as received.

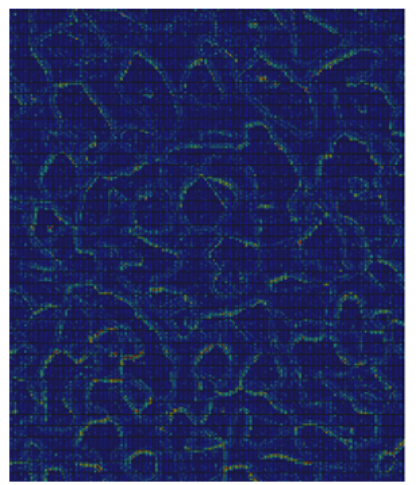

Figure 12: Energy 8 gray levels.

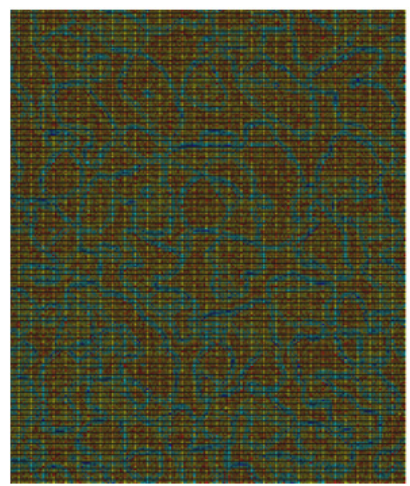

Figure 16: Mutual information 8 gray levels

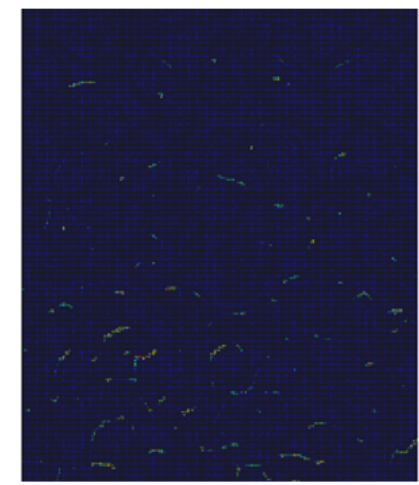

Figure 13: Energy 64 gray levels.

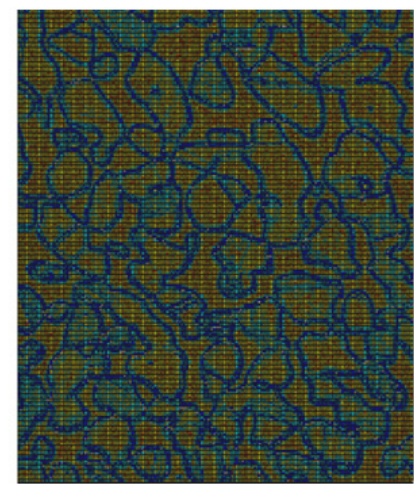

Figure 17: Mutual information 64 gray levels.

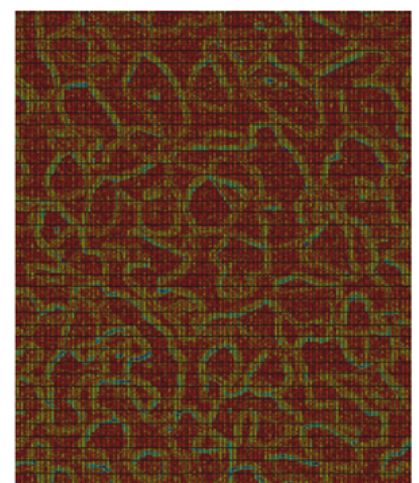

Figure 14: Entropy 8 gray levels.

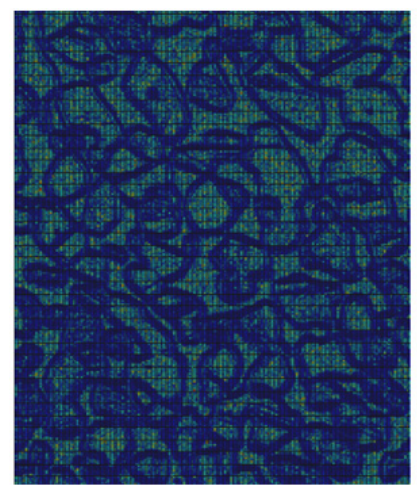

Figure 18: Contrast 8 gray levels.

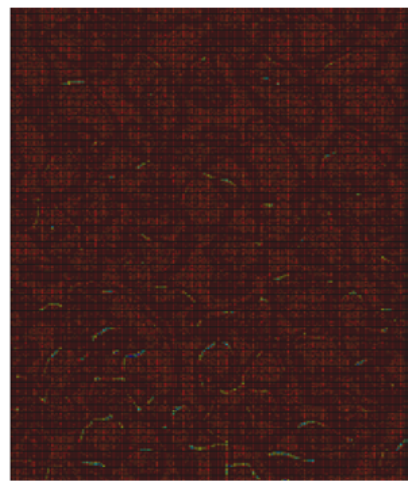

Figure 15: Entropy 64 gray levels.

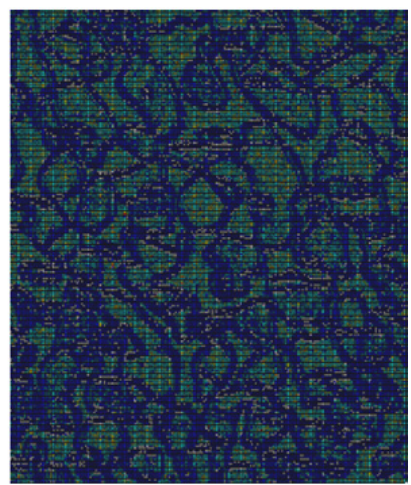

Figure 19: Contrast 64 gray levels.

\begin{tabular}{|c|c|c|c|c|}
\hline \multirow[t]{2}{*}{ INDICATOR } & \multicolumn{2}{|c|}{8 gray levels } & \multicolumn{2}{|c|}{64 gray levels } \\
\hline & Min & Max & Min & $\operatorname{Max}$ \\
\hline Energy & 0,1843 & 0,8906 & 0,1667 & 0,8361 \\
\hline Entropy & 0,7255 & 4,9477 & 1,0808 & 5,1699 \\
\hline Mutual Information & $-2,6769$ & $-0,0048$ & $-4,8366$ & $-0,1350$ \\
\hline Contrast & 0 & 14,1111 & 1,8611 & $1,0020 \mathrm{e}+03$ \\
\hline
\end{tabular}

Table 1: Minimum and maximum values assumed by the indicators. 


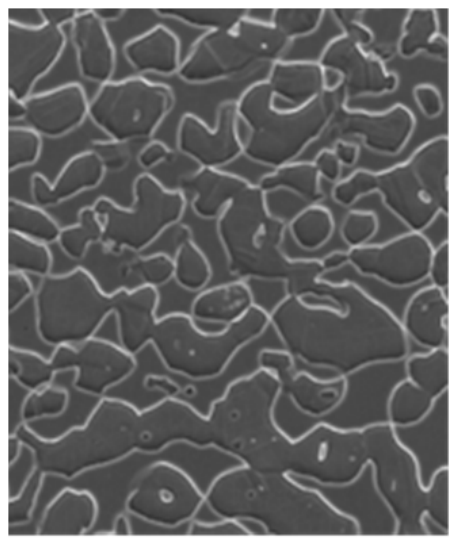

Figure 20: Duplex cold rolled.

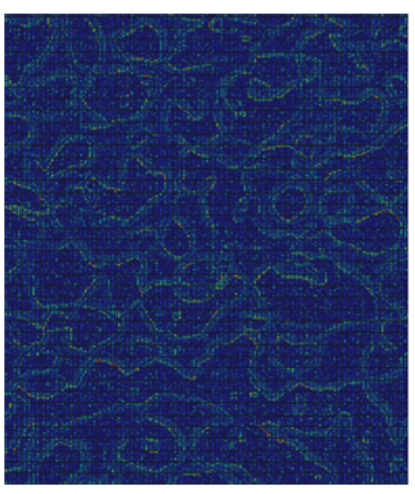

Figure 21: Energy 8 gray levels.

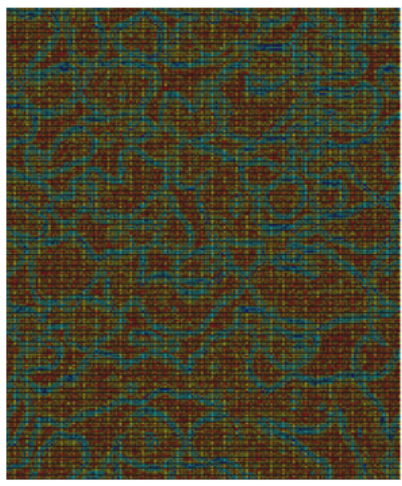

Figure 25: Mutual information 8 gray levels

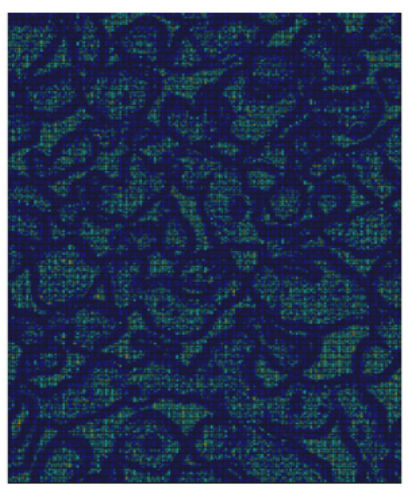

Figure 22: Energy 64 gray levels.

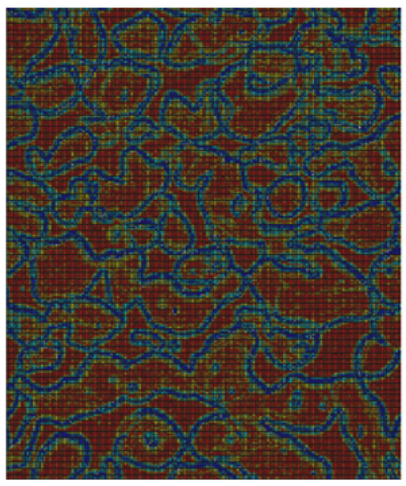

Figure 26: Mutual information 64 gray levels.

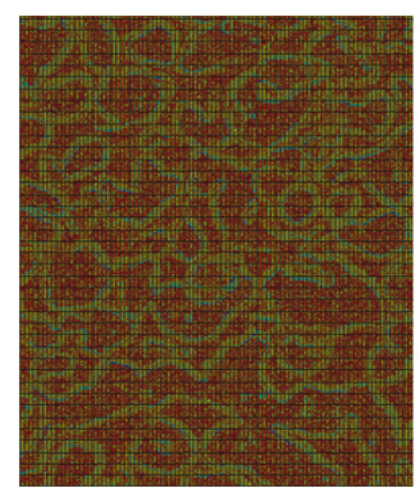

Figure 23: Entropy 8 gray levels.

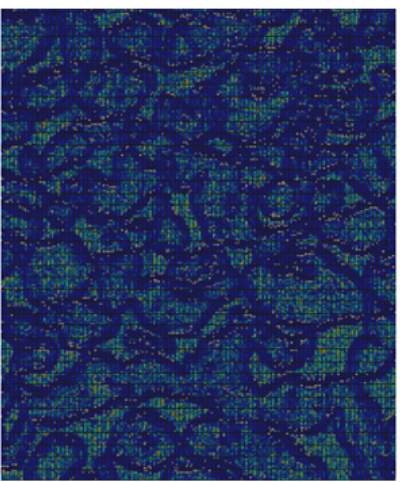

Figure 27: Contrast 8 gray levels.

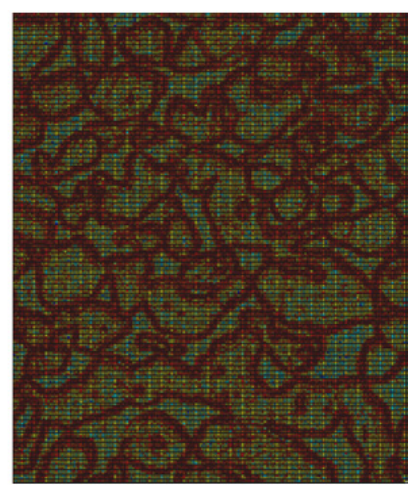

Figure 24: Entropy 64 gray levels.

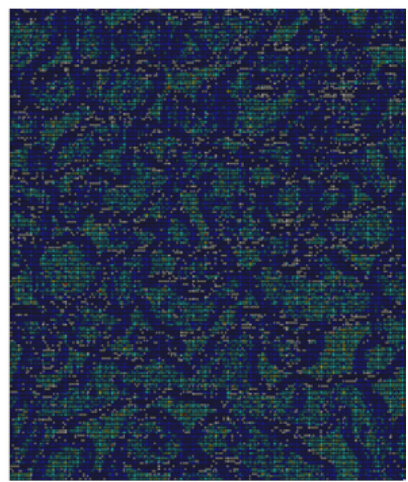

Figure 28: Contrast 64 gray levels.

\begin{tabular}{|c|c|c|c|c|}
\hline \multirow[t]{2}{*}{ INDICATOR } & \multicolumn{2}{|c|}{8 gray levels } & \multicolumn{2}{|c|}{64 gray levels } \\
\hline & Min & Max & Min & $\operatorname{Max}$ \\
\hline Energy & 0,1800 & 0,8099 & 0,1667 & 0,3849 \\
\hline Entropy & 1,2010 & 5,0033 & 3,4540 & 5,1699 \\
\hline Mutual Information & $-2,6615$ & $-0,1315$ & $-4,9267$ & $-0,2022$ \\
\hline Contrast & 0,0278 & 15,5278 & 1,6944 & $1,1013 e+03$ \\
\hline
\end{tabular}

Table 2: Minimum and maximum values assumed by the indicators. 


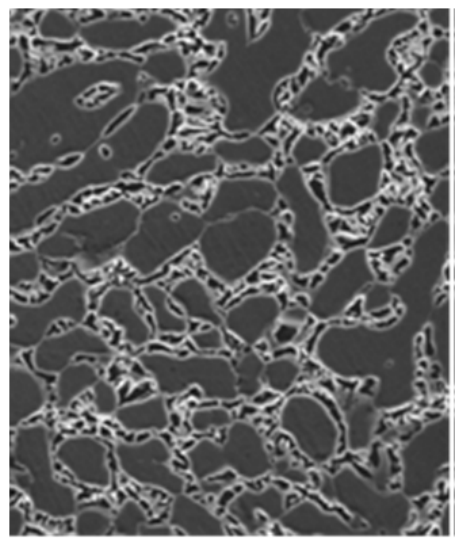

Figure 29: Duplex heat-treated.

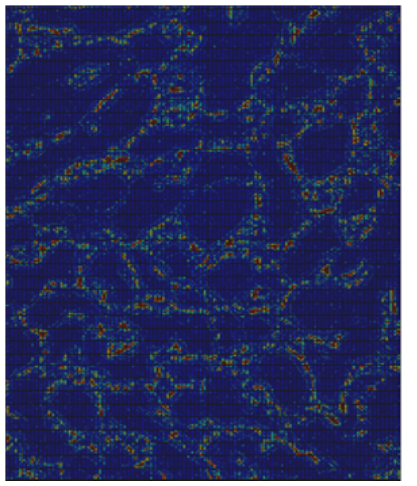

Figure 30: Energy 8 gray levels.

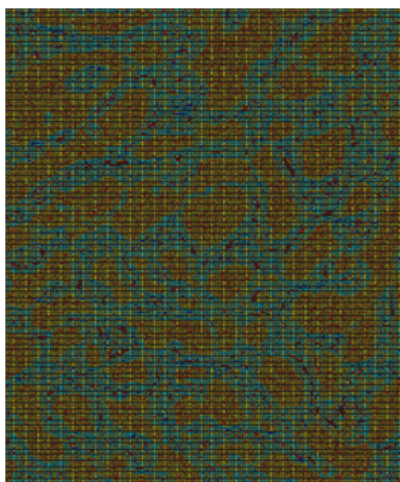

Figure 34: Mutual information 8 gray levels

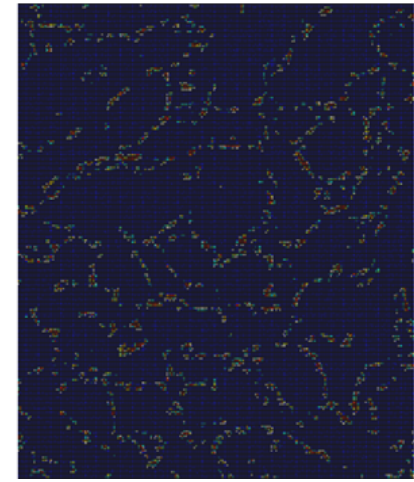

Figure 31: Energy 64 gray levels.

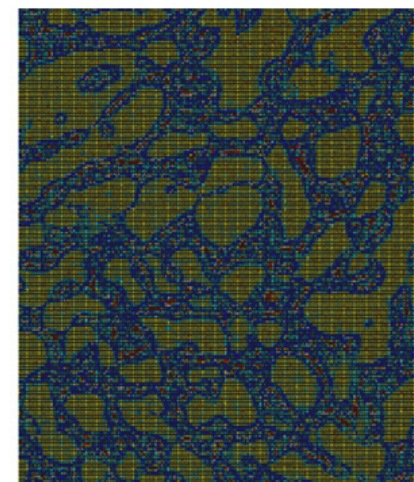

Figure 35: Mutual information 64 gray levels.

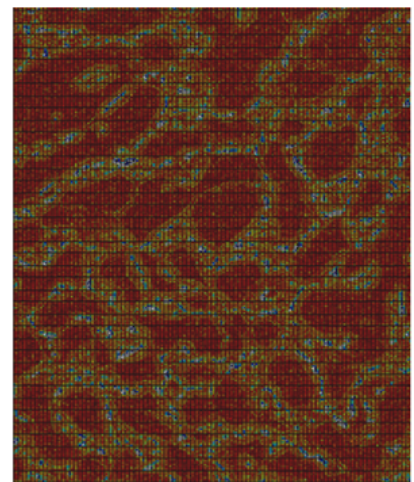

Figure 32: Entropy 8 gray levels.

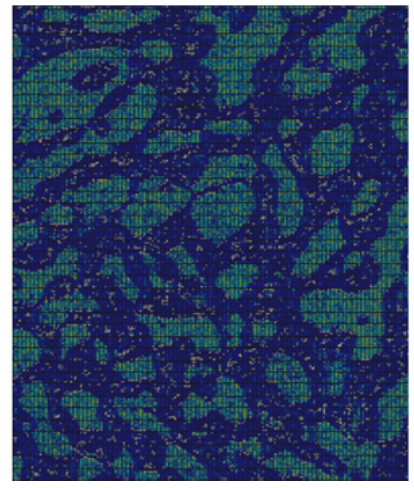

Figure 36: Contrast 8 gray levels.

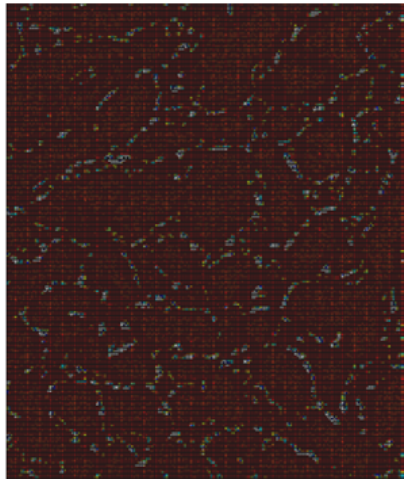

Figure 33: Entropy 64 gray levels.

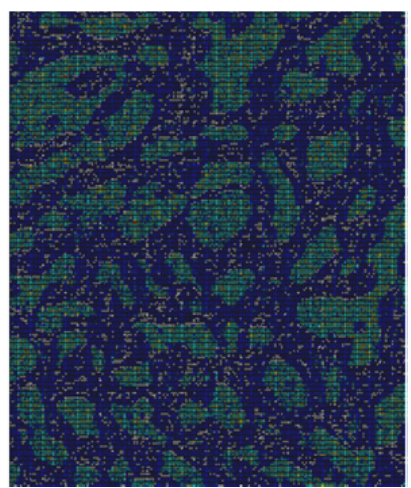

Figure 37: Contrast 64 gray levels.

\begin{tabular}{cccccc}
\hline INDICATOR & Min & gray levels & & 64 gray levels & Max \\
Energy & 0,1843 & Max & 1 & 0,1667 & 1 \\
Entropy & 0 & 4,9477 & 0 & 5,1699 \\
Mutual Information & $-2,6615$ & 0 & $-4,8712$ & 0 & $1,0430 \mathrm{e}+03$ \\
Contrast & 0 & 13,9444 & 0 & 0 \\
\hline
\end{tabular}

Table 3: Minimum and maximum values assumed by the indicators 


\section{CONCLUSIONS}

$\mathrm{T}$ he GLCM image analysis method is quite effective to classify materials textures. Encouraging results are achieved especially when the material has a structure that is fairly homogeneous i.e. when within the image there are patterns that repeat themselves continuously (grain size quite similar and uniformly distributed).

The image has been reduced to 8 and then to 64 gray levels to see if this largest number of gray affects the result.

The first thing you notice is the visual correspondence between the indicator map and the real image.

Regarding Entropy and Energy Indicators, 8 levels are sufficient to give information, thus saving time due to the increased speed of calculation.

Maps in 64 levels are more difficult to interpret probably because a high level of gray makes less noticeable changes in texture.

Contrast Indicator has a greater clarity of interpretation in 8 levels, although in 64 it has the maximum values very high, due to the higher background noise provided by the large number of grays.

Mutual Information provides a better result in 64 levels, because the distribution of numerical values provides a map closer to the duplex morphology. In fact we see from the tables that the values of MI in 64 levels are higher than in 8 levels, this corresponds to an emphasis of the grain boundary.

The Entropy indicator is appropriate to identify the grain boundaries and presupposes the presence of some geminates. The Contrast indicator, although it is able to detect the continuity of tones, doesn't permit identification of the components of the duplex morphology. The best information obtained by the Energy indicator is instead the presence of impurities. Mutual Information emphasizes the presence of impurities and minimizes the tool marks resulting from the polishing.

Although these statistical indicators based on the GLCM are quite suitable for image analysis in the classification of the texture, they give rise to an effect in the final image, consisting in the thickening of the border that increases with the size of the window with which is built GLCM [8]. It should be noted that the computation time of the indicators increases exponentially with the increase of the window's size and the number of gray levels of the image. There is no single method that can actually give an aid in the analysis and discrimination of all textures. Choice must be made each time from the starting image.

In the calculation process a detection window is used and the characteristics obtained are assigned to the pixel which is located in central position and then scroll of one unit. A consequence of this modus operandi is that there are pixels whose mutual relations are not evaluated and in correspondence of them the value of adjacent pixels is assigned. This is the reason why we have the thickening of the border.

\section{REFERENCES}

[1] ASM Handbook, Volume 01 - Properties And Selection: Irons Steels And High Performance Alloys

[2] M. Boniardi, F. D’Errico, C. Mapelli, Microstruttura, trattamenti termici e proprietà meccaniche degli acciai inossidabili bifasici, Dip. di Meccanica, Politecnico di Milano.

[3] R. M. Haralick, K. Shanmugam, I. Dinstein, IEEE Transactions on Systems, Man and Cybernetics, 3 (1973) 610.

[4] R. M. Haralick, In: Proceedings of the IEEE, 67 (1979).

[5] M. Hall-Beyer: "Glcm texture: a tutorial" - www.ucalgary.ca \mhallbey (2000).

[6] A. Umeda, J. Sugimura, Y. Yamamoto, Wear 216 (1998) 220.

[7] A. Gallo: "Metallografia quantitativa" - Fondazione politecnica per il mezzogiorno d'Italia - Quaderno 124 - Giannini, Napoli, (1979).

[8] S. J. Yin, X. L. Chen, In: 9th International Conference on Geocomputation, National University of Ireland, Maynooth, Eire (2007). 